\title{
Um Modelo Qualitativo para Explicar a Influência da Polaridade na Taxa de Fusão no Processo MIG/MAG
}

\author{
(A Qualitative Model to Explain the Polarity Influence on the Fusion Rate in the MIG/MAG Process)
}

\author{
Daniel Souza ${ }^{1}$, André Alves de Resende ${ }^{1}$, Américo Scotti ${ }^{1}$ \\ ${ }^{1}$ Universidade Federal de Uberlândia, Faculdade de Engenharia Mecânica, Centro para Pesquisa e Desenvolvimento de Processos \\ de Soldagem, Bloco 5H, Av. João Naves de Ávila, 2121, CEP 38400 902,Uberlândia-MG, dsouza@mecanica.ufu.br, aaresende@ \\ gmail.com, ascotti@mecanica.ufu.br
}

\begin{abstract}
Resumo
Uma das principais vantagens do processo MIG/MAG é a alta produtividade. Na maioria das aplicações utiliza-se a polaridade positiva, devido a maior estabilidade do arco, geração de menos respingos e formação de cordões com geometria adequada. No entanto, em algumas aplicações, se demanda uma maior capacidade de produção do que a que o MIG/MAG convencional oferece. Na literatura é citado que a polaridade negativa proporciona maior taxa de fusão do que a positiva, apesar de levar à grande produção de respingos e formação inadequada do cordão. Lamentavelmente, não há muita informação disponível sobre os efeitos das variáveis do processo nessa polaridade, tão pouco justificativas sobre tal. Assim, neste trabalho se procurou entender a razão para que uma maior taxa de deposição aconteça na polaridade negativa, assim como o respectivo efeito sobre a geometria dos cordões. Para isto, foram realizadas soldagens MIG/MAG comparativas na polaridade positiva e negativa, usando duas composições de gases de proteção em dois valores de corrente. O modo de transferência e o comportamento do arco foram analisados por perfilografia sincronizada. $O$ perfil geométrico do cordão foi avaliado por meio de procedimentos metalográficos. A partir dos resultados, que discordam em parte da literatura corrente, foi observado que tanto o modo de transferência como a morfologia e aspecto do cordão são dependentes da composição do gás de proteção. Para explicar os fenômenos inerentes à maior taxa de fusão do arame em CC-, sugere-se que o fato de o arco escalar as laterais do arame nesta polaridade seja o fator governante.
\end{abstract}

Palavras Chave: Soldagem, MIG/MAG; transferência metálica; polaridade negativa

\begin{abstract}
One of the main advantages of the MIG/MAG process is its high productivity. Usually, the positive polarity is employed, due to a higher arc stability, less spattering and adequate bead profiles. However, a higher production capacity than observed in the conventional MIG/MAG process has been demanded for some applications. In the current literature related to MIG/MAG, it is mentioned that the negative polarity provides a higher fusion rate than the positive one, despite of leading to higher spattering and bead misshapen. Unfortunately, there is no much information available about the effects of process variables in this polarity, as much as concerning justifications. Thus, in this work was attempted to understand the reasons for a higher fusion rate at CC-, as well as the respective effect on the bead geometry. For that, MIG/MAG comparative welds were carried out at both polarities, using two shielding gas blends and two current values. The transfer mode and arc behavior were assessed by synchronized shadowgrafy technique. The bead geometry was evaluated through metallographic procedures. From the results, which does not agree entirely with the current literature, it was observed that as much the transfer mode as the bead morphology and finish depend on the shielding gas composition. To explain the phenomena inherent to a higher fusion rate at CC-, it is claimed that the fact of the arc to climb the wire surface at this polarity is the governing factor.
\end{abstract}

Keywords: Welding, MIG/MAG; metal transfer; negative polarity

\section{Introdução}

O processo de soldagem MIG/MAG (também conhecido como GMAW - (Gas Metal Arc Welding) é definido pelo "Welding Handbook" da American Welding Society [1] como um processo de soldagem a arco que usa um arco entre um arame-eletrodo de alimentação contínua e uma poça de fusão.

(Recebido em 17/03/2009; Texto Final em 23/05/2009).
É usado com uma proteção externa de gás sem a aplicação de pressão. Apesar da aparente simplicidade da definição, existem vários fatores que afetam a operacionalidade desse processo, como, entre outros, as composições químicas dos arameseletrodo e dos gases de proteção. Esses fatores, por sua vez, afetam diretamente as propriedades mecânicas e metalúrgicas do metal da solda, os modos de transferência metálica, etc.

A soldagem pelo processo MIG/MAG convencional normalmente é utilizada em CC+ (com o eletrodo ligado ao pólo positivo e a peça ao pólo negativo, também conhecido como polaridade inversa). De acordo com a literatura corrente, nesta 
configuração, consegue-se grande profundidade de penetração, boa estabilidade do arco e da transferência, quantidade de respingos relativamente pequena, além da possibilidade de soldar com os diferentes modos de transferência metálica (curtocircuito, globular e as várias formas da transferência goticular -"spray"). A soldagem com o eletrodo ligado ao pólo negativo (CC-), também conhecida como polaridade direta, é dita ter características de baixa penetração, alta taxa de fusão de arame para uma dada corrente em comparação à soldagem com $\mathrm{CC}+, \mathrm{a}$ qual leva a uma menor quantidade de calor transferido à peça e quantidade de respingos relativamente alta.

A soldagem em CC-, segundo Talkington [2], é limitada geralmente à transferência globular e é pouco usada na prática porque o arco resultante é instável e os respingos produzidos são indesejáveis. Ainda, segundo este pesquisador, o maior problema inerente à soldagem em CC- é a força catódica repulsiva que atua na extremidade fundida do elétrodo, já que os elétrons são emitidos do eletrodo e transferidos à superfície de trabalho (peça). Lancaster [3] descreve estas forças de reação como a principal causa para gotas assimetricamente repelidas durante MIG/MAG em CC-. Ele também descreve o posicionamento instável do ponto catódico que forma na extremidade da gota fundida; durante a soldagem, o ponto catódico é identificado por uma intensa concentração de luz emitida de uma área localizada da gota que se destaca em meio ao arco. O ponto catódico pode ser observado "dançando" ao redor de uma gota grande no momento em que ela esta sendo assimetricamente repelida, como ilustra a Figura 1.

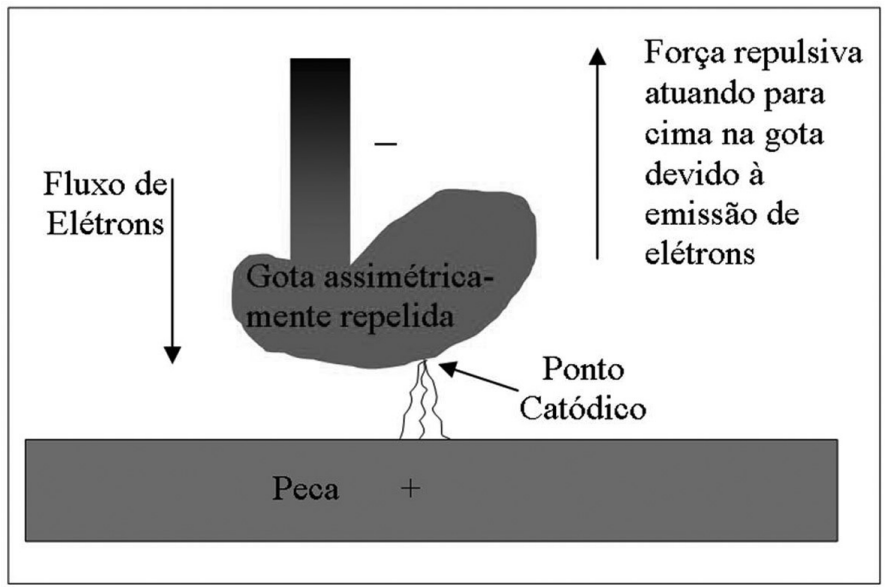

Figura 1. Gota assimetricamente repelida e ponto catódico. Adaptado de Lancaster [3].

Scotti [4], em uma revisão sobre transferência metálica, cita que além do efeito da polaridade, gotas repelidas acontecem devido ao tipo de gás de proteção, mesmo em $\mathrm{CC}+$. Inicialmente citando Jönsson et al. [5], o efeito do gás He como proteção sobre este fenômeno é destacado; gotas deformadas são formadas devido a um fluxo de gás (jato de plasma) na direção peça-gota. Este fluxo mássico, por sua vez, é causado por forças eletromagnéticas (gradiente de forças de Lorentz) geradas próximo à superfície do cátodo, em função de uma convergência marcante da corrente na vizinhança da poça (ponto catódico muito pequeno). Mas este autor também cita que mesmo com outros gases gotas podem se tornar repelidas em $\mathrm{CC}+$. Ushio et al. [6], em um estudo sobre MIG/MAG com um arameeletrodo de aço de 1,2 mm, observaram transferências regulares somente nos modos globular e spray em suas respectivas escalas operacionais, quando uma mistura $0-10 \% \mathrm{CO}_{2}-\mathrm{Ar}$ foi usada. A transferência mudou predominantemente para repelida (flexionada) nas modalidades globular e goticular quando o índice do $\mathrm{CO}_{2}$ excedeu 10\%. Rhee e Kannatey-Asibu Jr.'s [7] reportaram gotas repelidas com proteção de $100 \% \mathrm{CO}_{2}$, efeito associado com forças eletromagnéticas e de fumos. Finalmente, Scotti [4] cita ainda a tendência para transferência globular repelida ser esperada decrescer com o aumento da corrente, mas que até aquela época não havia prova desta hipótese.

Apesar das características indesejáveis de se soldar em CC-, esta abordagem oferece algumas características úteis para a soldagem GMAW. O principal beneficio da CC- seria o balanço reverso de calor produzido no arco. Segundo Talkington [2], a característica da soldagem GMAW em CC+é que aproximadamente $30 \%$ do calor gerado no arco é transferido ao eletrodo e o restante (aproximadamente 70\%) ao metal de base. Este balanço energético é reverso para soldagem na $\mathrm{CC}$-, aproximadamente $30 \%$ da energia é transferida ao metal de base e $70 \%$ para o eletrodo. Alguns pesquisadores, como Lancaster [3], estudaram os vários fatores que afetam a velocidade de fusão e tem mostrado que maiores velocidades de fusão (maior volume depositado por unidade de comprimento de solda) e menores penetrações são obtidas em CC- comparada a $\mathrm{CC}+$. O cordão assume um formato extremamente convexo, não adequado para soldagem. A Figura 2 resume as diferenças relativas esperadas (baseado na descrição de ambos os pesquisadores) entre os perfis dos cordões de solda resultantes da soldagem MIG/MAG em $\mathrm{CC}$ - e $\mathrm{CC}+$.

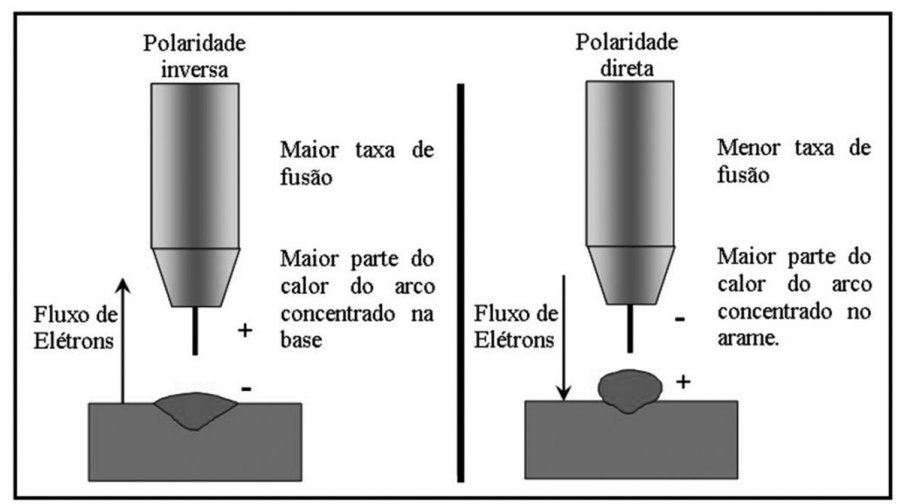

Figura 2. Características do cordão de solda para soldagem na CC- e inversa (adaptado de Talkington [2]).

Pelo exposto, parece ser consenso que a soldagem em CCproduz maior taxa fusão, mas com uma transferência globular repelida e um cordão extremante convexo e de muito baixa penetração. Entretanto, experiências exploratórias no Centro para Pesquisa e Desenvolvimento de Processos de Soldagem (LAPROSOLDA) da Universidade Federal de Uberlândia deram evidência de que nem sempre $\mathrm{CC}$ - conduzia à baixa penetração ou que a transferência era do tipo globular repulsiva. Além disto, outros experimentos mostraram que arames aditivados fazem 
com que a taxa de fusão em ambas as polaridades se igualem. Desta forma, as explicações acima citadas da literatura sobre a razão para a maior taxa de fusão baseada em distribuição térmica nas duas polaridades ou que CC-provoca transferência repelidas são pelo menos não genéricas. Assim, há ainda uma lacuna na bibliografia sobre o real papel da polaridade sobre a transferência metálica e sobre a geometria do cordão no processo MIG/MAG. Desta forma, neste trabalho objetiva-se ampliar os conhecimentos sobre estes aspectos, visando entender melhor os fenômenos que ocorrem durante a soldagem MIG/MAG em CC-.

\section{Procedimentos Experimentais}

Um total de oito experimentos foi realizado visando verificar as influências da composição do gás proteção, do nível de corrente e da polaridade (CC+ e CC-) sobre os modos de transferência metálica na soldagem MIG/MAG. Na realização de tais experimentos, foram utilizadas uma tocha comercial refrigerada a água e uma fonte eletrônica multiprocesso do tipo chaveada no secundário. A fonte foi programada para trabalhar no modo MIG/MAG corrente constante, para se garantir o mesmo valor de corrente em todos os experimentos comparativos. Neste modo, regula-se o valor da corrente e da velocidade de alimentação, sendo a tensão a resultante da carga (caracterizada por cada arco). Para procurar uma condição similar de trabalho quando se usava CC+ ou CC-, a velocidade de alimentação em cada caso era aumentada até se iniciar curtos-circuitos. O ponto de trabalho era, então, definido com a velocidade de alimentação do instante anterior ao início dos curtos. Chama-se atenção de que para pequenos comprimentos de cordão e nas condições laboratoriais impostas, não se verificou variação significativa do comprimento médio do arco durante as soldagens. O uso de um controle de comprimento do arco foi, assim, descartado, pois o mesmo seria intruso ao fenômeno em estudo.

O arame-eletrodo utilizado no ensaio foi da classe AWS ER70S-6, de diâmetro 1,2 mm. Utilizou-se como gás de proteção as misturas de $92 \% \mathrm{Ar}+8 \% \mathrm{CO}_{2}$ e $98 \% \mathrm{Ar}+2 \% \mathrm{O}_{2}$. A vazão utilizada em todos os testes foi de $13 \mathrm{l} / \mathrm{mim}$. Para efeito de comparação, todos os ensaios foram realizados como simples deposição sobre chapa. As chapas de teste foram confeccionadas a partir de chapas de aço carbono ABNT 1020 de dimensões 200 x 25,4 x 9,5 mm.

A Tabela 1 mostra os parâmetros regulados para cada teste. Os Testes 1 a 4 foram realizados para verificar a influência do gás de proteção, enquanto os Testes 5 e 6 para verificar a influência da corrente no modo de transferência, comparativamente aos Testes 1 e 2 . Os Testes 7 e 8, comparados respectivamente com os Testes 1 e 4, foram feitos para verificar o efeito da polaridade, usando a mesma relação velocidade de alimentação/velocidade de soldagem, com o intuito de se obter uma mesma quantidade de material depositado por unidade de comprimento e se comparar os perfis dos cordões de solda tanto em CC+ como em CC-.

Tabela 1. Parâmetros de regulagem para os Testes.

\begin{tabular}{cccccccc}
\hline Teste & Gás de Proteção & Polaridade & $\begin{array}{c}\text { Velocidade de } \\
\text { Alimentação } \\
(\mathrm{m} / \mathrm{min})\end{array}$ & $\begin{array}{c}\text { Velocidade } \\
\text { Soldagem } \\
(\mathrm{cm} / \mathrm{min})\end{array}$ & $\begin{array}{c}\text { DBCP } \\
(\mathrm{mm})\end{array}$ & $\begin{array}{c}\text { Vazão de } \\
\text { Gás } \\
(\mathrm{l} / \mathrm{min})\end{array}$ & $\begin{array}{c}\text { Corrente } \\
\text { Referência } \\
(\mathrm{A})\end{array}$ \\
\hline 1 & $\mathrm{Ar}+2 \% \mathrm{O}_{2}$ & $\mathrm{CC}-$ & 11,7 & 34,8 & 20 & 13 & 250 \\
2 & $\mathrm{Ar}+2 \% \mathrm{O}_{2}$ & $\mathrm{CC}+$ & 7,2 & 34,8 & 20 & 13 & 250 \\
4 & $\mathrm{Ar}+18 \% \mathrm{CO}_{2}$ & $\mathrm{CC}-$ & 9,8 & 34,8 & 20 & 13 & 250 \\
3 & $\mathrm{Ar}+18 \% \mathrm{CO}_{2}$ & $\mathrm{CC}+$ & 7,0 & 34,8 & 20 & 13 & 250 \\
6 & $\mathrm{Ar}+2 \% \mathrm{O}_{2}$ & $\mathrm{CC}-$ & 7,2 & 34,8 & 20 & 13 & 150 \\
5 & $\mathrm{Ar}+2 \% \mathrm{O}_{2}$ & $\mathrm{CC}+$ & 3,2 & 34,8 & 20 & 13 & 150 \\
7 & $\mathrm{Ar}+2 \% \mathrm{O}_{2}$ & $\mathrm{CC}+$ & 7,2 & 21,5 & 20 & 13 & 250 \\
8 & $\mathrm{Ar}+18 \% \mathrm{CO}_{2}$ & $\mathrm{CC}+$ & 7,0 & 25,0 & 20 & 13 & 250 \\
\hline
\end{tabular}

Para visualização da transferência metálica e dos fenômenos correlatos, foi utilizada a técnica de perfilografia com filmagem digital a alta velocidade com 2000 qps e luz de fundo de laser 632,2 $\mu \mathrm{m}$ [8]. Em soldagem, o termo "perfilografia" é proposto para se referenciar à formação da sombra projetada de vários elementos (tocha, eletrodo, gotas, cordão e chapa) em um filme fotográfico ou diretamente sobre a lente de uma filmadora, técnica também conhecida como "Back-lighting" (Figura 3). $\mathrm{O}$ arranjo experimental foi montado de acordo com a Figura 4. Para se permitir visualizar também o arco, foi preciso penalizar a definição das gotas em formação e transferência, através da intensidade dos filtros ópticos usados. Para correlacionar a variação da tensão e corrente com a formação e destacamento das gotas, os sinais elétricos foram sincronizados com os quadros de filmagem, pela técnica descrita em outro artigo [9]. O efeito sobre a geometria foi estudado através de técnicas convencionais de macrografia, sobre seções transversais tiradas das placas de teste soldadas.

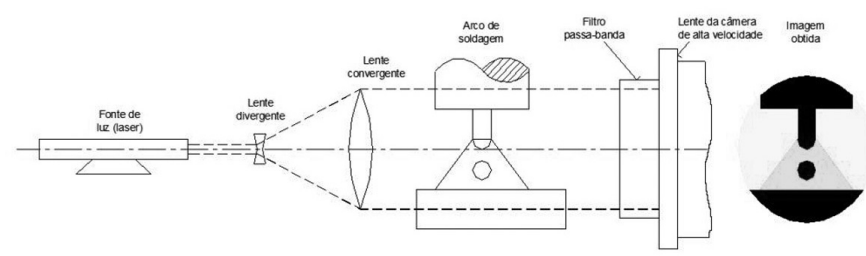

Figura 3. Princípio da Perfilografia aplicada à soldagem [10]. 
Tabela 2. Valores monitorados durante os Testes.

\begin{tabular}{ccccc}
\hline \multirow{2}{*}{ Teste } & \multicolumn{2}{c}{ Tensão } & \multicolumn{2}{c}{ Corrente } \\
\cline { 2 - 5 } & Média & RMS & Média & RMS \\
\hline 1 & 32,4 & 32,7 & 249 & 249 \\
2 & 28,5 & 28,7 & 250 & 250 \\
4 & 43,4 & 43,6 & 255 & 255 \\
3 & 34,7 & 34,9 & 251 & 251 \\
6 & 24,0 & 24,4 & 155 & 158 \\
5 & 23,5 & 24,3 & 149 & 149 \\
7 & 32,3 & 32,6 & 250 & 250 \\
8 & 43,0 & 43,2 & 251 & 251 \\
\hline
\end{tabular}

\section{Resultados e Discussão}

Na Tabela 2 têm-se os valores de tensão e corrente monitorados durante os testes, enquanto o tipo de transferência e o comportamento dos sinais de tensão e corrente dos Testes 1 a 6 são apresentados nas Figuras 5 a 10. Inicialmente foi observado que, para mesma corrente, o uso de CC- leva a uma maior taxa de fusão, quantificado pela velocidade de alimentação regulada para ter soldas com estabilidade para as dadas correntes (Tabela 1). Nestas condições de soldagem, obteve-se transferência globular (baixa corrente) e goticular (maior corrente) em CC-, o que contraria o mencionado na literatura principalmente para gases ricos em $\mathrm{CO}_{2}$.

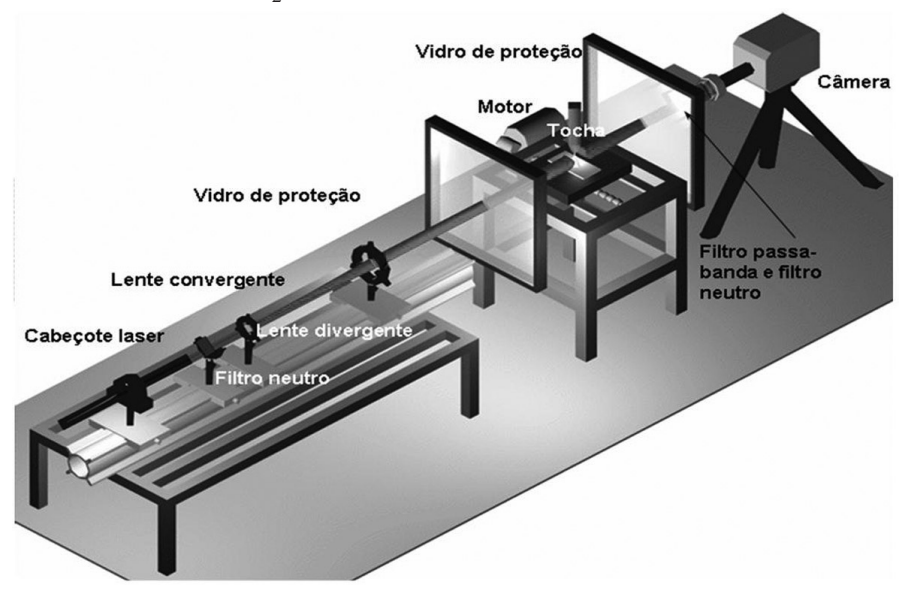

Figura 4. Detalhe do sistema laser-óptico utilizado [10].

Ao se observar os gráficos associados à soldagem em $\mathrm{CC}+$, para as duas misturas de gás e valores de corrente utilizados nos testes, podem ser averiguado um menor comprimento de arco em relação à soldagem em CC- (a medição do comprimento do arco não foi realizada, somente uma avaliação visual, até pela dificuldade em definir o que seria comprimento de arco, que para as duas polaridades e diferentes gases possuem características de acoplamento e emissão luminosa distintos). Verificou-se uma tendência do arco "escalar" no arame, provocando um aumento no comprimento de arco. Esse efeito é mais pronunciado quando se usa um pequeno percentual de oxigênio como gás ativo. Considerando, além destes resultados, os conceitos de emissão por campo (ou emissão de cátodo frio) e o conhecido fenômeno de limpeza catódica em soldagem TIG CA de alumínio ("sputtering zone"), este comportamento passou a ser considerado no presente artigo como o principal responsável pelas maiores taxas de consumo de material de adição em relação à soldagem em CC+ (ao invés de se considerar apenas que na conexão anódica se gera mais calor do que na catódica). Assim, um modelo proposto pelos autores para explicar a maior taxa de fusão em CC- seria a maior eficiência térmica conseguida com o arco atingido uma maior área da ponta do eletrodo em procura de óxidos para emissão por campo.

Esse comportamento do arco na soldagem em CC- explica algumas das particularidades desse processo, como a maior instabilidade do arco que conseqüentemente provoca maiores variações nos sinais de corrente e tensão (ver Figuras 5 a 10) e o aumento das quantidades de respingos observados ao se soldar nessa condição. A instabilidade do arco e os respingos já foram anteriormente observados por Talkington [2], que cita também que a transferência nesta polaridade é normalmente limitada ao modo globular. Porém essa afirmação só se confirmou para o Teste $6\left(\mathrm{Ar}+2 \% \mathrm{O}_{2}\right)$, no qual a soldagem foi realizada em nível baixo de corrente (150 A). Já para os Testes $1\left(\mathrm{Ar}+2 \% \mathrm{O}_{2}\right)$ e $4\left(\mathrm{Ar}+18 \% \mathrm{CO}_{2}\right)$, realizados com nível mais alto de corrente (250 A), mostraram que tal afirmação nem sempre é valida, já que foi observada transferência no modo goticular.

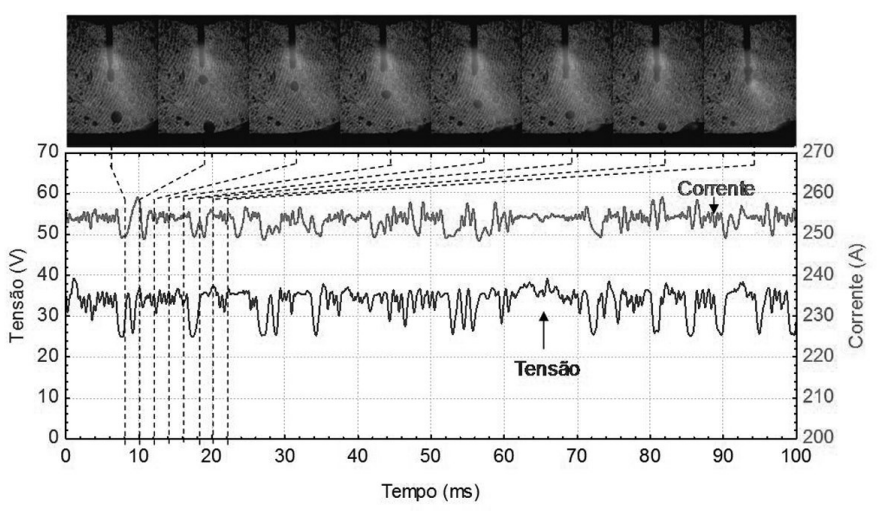

Figura 5. Comportamento temporal dos sinais de tensão e corrente com ilustração sincronizada da formação do arco durante as gotas em crescimento e destacamento para o Teste 1 $\left(\mathrm{Ar}+2 \% \mathrm{O}_{2} ; 250 \mathrm{~A} ; \mathrm{CC}-\right)$. 


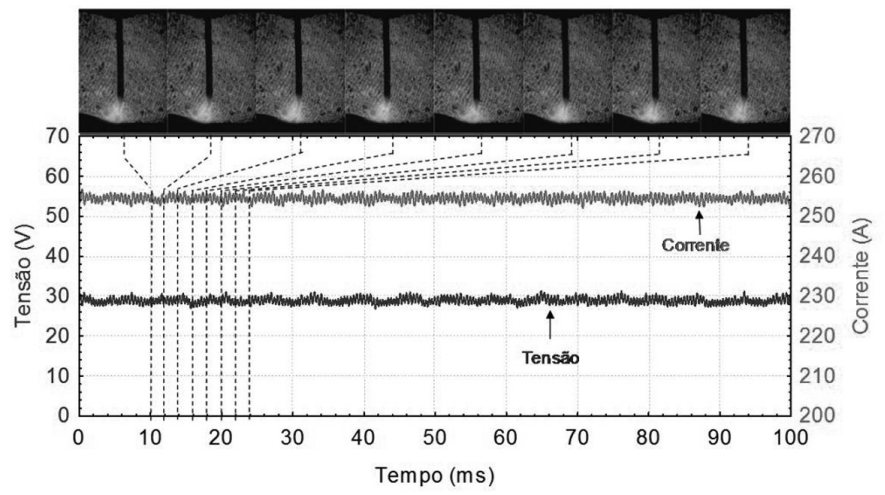

Figura 6. Comportamento temporal dos sinais de tensão e corrente com ilustração sincronizada da formação do arco durante as gotas em crescimento e destacamento para o Teste 2 $(\mathrm{Ar}+2 \% \mathrm{O} 2 ; 250 \mathrm{~A} ; \mathrm{CC}+)$.

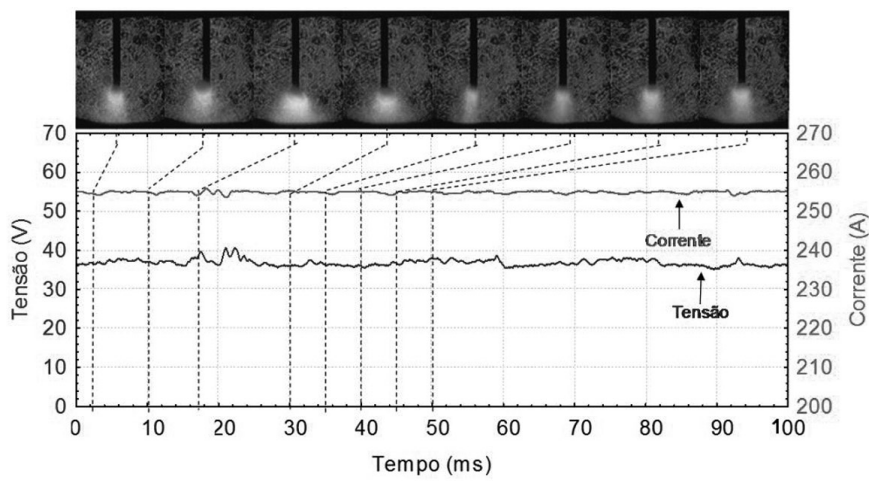

Figura 7. Comportamento temporal dos sinais de tensão e corrente com ilustração sincronizada da formação do arco durante as gotas em crescimento e destacamento para o Teste 3 $\left(\mathrm{Ar}+18 \% \mathrm{CO}_{2} ; 250 \mathrm{~A} ; \mathrm{CC}+\right)$.

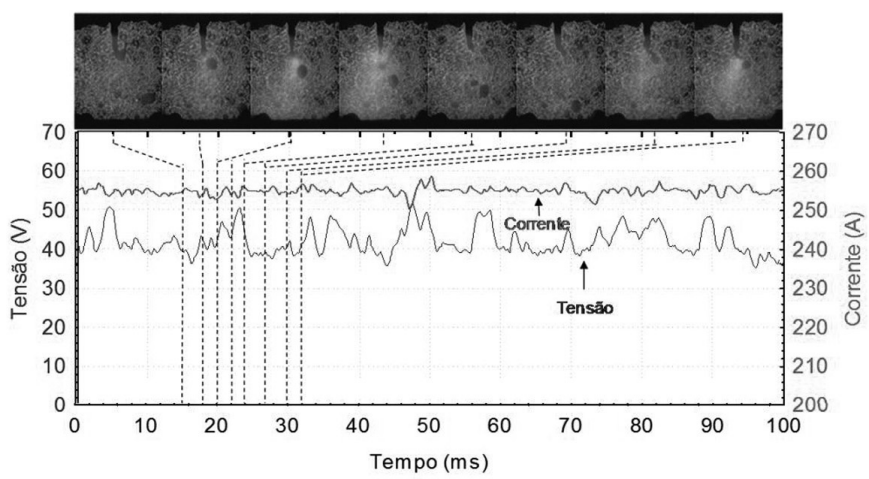

Figura 8. Comportamento temporal dos sinais de tensão e corrente com ilustração sincronizada da formação do arco durante as gotas em crescimento e destacamento para o Teste 4 $\left(\mathrm{Ar}+18 \% \mathrm{CO}_{2} ; 250 \mathrm{~A} ; \mathrm{CC}-\right)$.

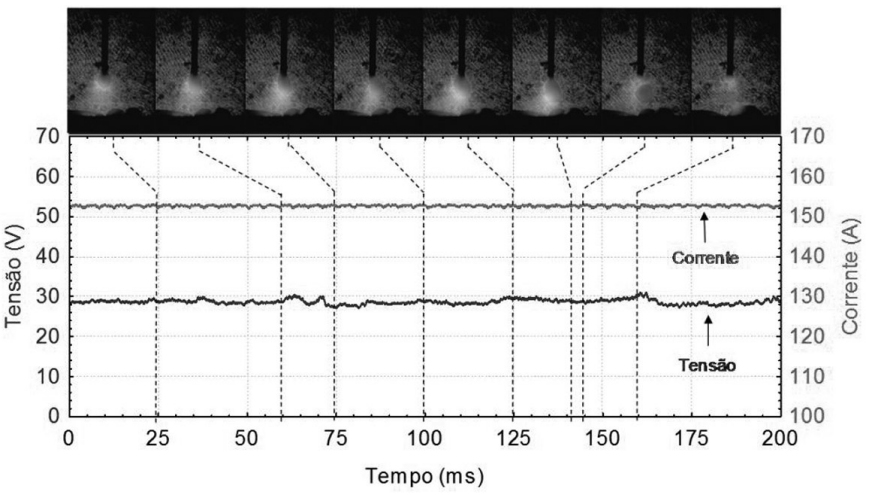

Figura 9. Comportamento temporal dos sinais de tensão e corrente com ilustração sincronizada da formação do arco durante as gotas em crescimento e destacamento para o Teste 5 $\left(\mathrm{Ar}+2 \% \mathrm{O}_{2} ; 150 \mathrm{~A} ; \mathrm{CC}+\right)$

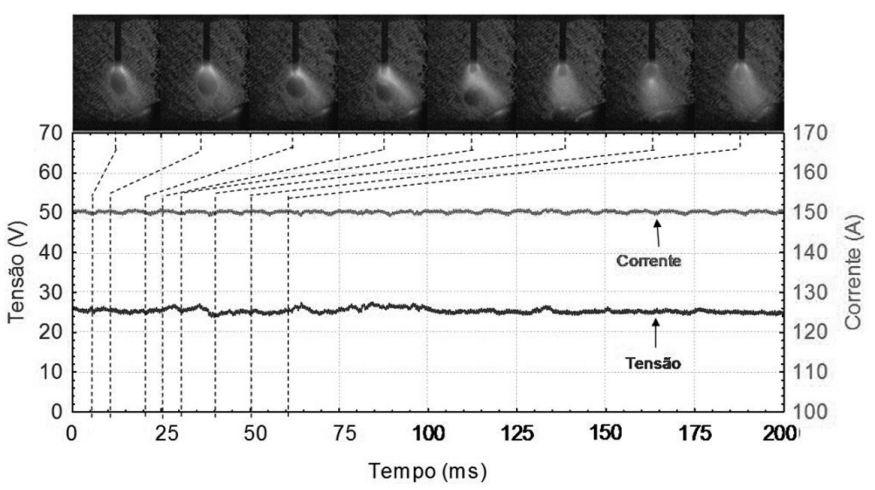

Figura 10. Comportamento temporal dos sinais de tensão e corrente com ilustração sincronizada da formação do arco durante as gotas em crescimento e destacamento para o Teste 6 $\left(\mathrm{Ar}+2 \% \mathrm{O}_{2} ; 150 \mathrm{~A} ; \mathrm{CC}-\right)$.

A Figura 11 mostra os aspectos dos cordões de solda e suas respectivas seções transversais reveladas por ataque macrográfico, enquanto a Tabela 3 apresenta os valores dos parâmetros geométricos medidos. Analisando-se inicialmente o efeito do gás de proteção (veja as características resultantes dos Testes 1 x 4 e 2 x 3 , todos à $250 \mathrm{~A}$ ), verifica-se que a proteção com $18 \% \mathrm{CO}_{2}$ fez aumentar a largura do cordão, deixando o cordão menos convexo, mas proporcionou cordões com piores aspectos. É importante ressaltar que a proteção com $18 \% \mathrm{CO}_{2}$ também fez reduzir a taxa de fusão do arame-eletrodo (ou velocidade de alimentação) em relação às soldagens com $\mathrm{Ar}+2 \% \mathrm{O}_{2}$ em mesmo nível de corrente (este aspecto e a redução da convexidade é mais evidente em CC-), o que deveria favorecer a redução da largura. $\mathrm{O}$ uso da proteção com $\mathrm{Ar}+18 \% \mathrm{CO}_{2}$ também fez aumentar a área fundida (maior eficiência de fusão do material de base) nas duas polaridades. Porém, quanto à penetração e perfil do cordão, o efeito do gás de proteção mostrou depender da polaridade. Em CC+ (Testes 2 x 3), a influência da composição do gás de proteção parece ser minimizada por outros efeitos, já que praticamente não se nota diferenças na profundidade de penetração e no perfil do cordão. Já se torna evidente em CC- (Testes 1 x 4), quando a penetração se reduz em praticamente $100 \%$ quando o gás passa de $\mathrm{Ar}+2 \% \mathrm{O}_{2}$ para $\mathrm{Ar}+18 \% \mathrm{CO}_{2}$. 
Tabela 3. Parâmetros geométricos medidos nas seções transversais das placas de teste.

\begin{tabular}{ccccc}
\hline Teste & $\begin{array}{c}\text { Área fundida } \\
\left(\mathrm{mm}^{2}\right)\end{array}$ & $\begin{array}{c}\text { Largura } \\
(\mathrm{mm})\end{array}$ & $\begin{array}{c}\text { Altura do reforço } \\
(\mathrm{mm})\end{array}$ & $\begin{array}{c}\text { Penetração } \\
(\mathrm{mm})\end{array}$ \\
\hline 1 & 7,2 & 8,0 & 5,3 & 2,0 \\
2 & 16,8 & 9,4 & 3,0 & 3,3 \\
4 & 9,8 & 12,4 & 3,0 & 1,1 \\
3 & 19,2 & 11,7 & 3,0 & 3,2 \\
6 & 0,8 & 6,3 & 4,5 & 0,4 \\
5 & 4,1 & 6,5 & 2,2 & 1,2 \\
7 & 26,7 & 13,6 & 3,8 & 3,6 \\
8 & 30,3 & 17,1 & 3,1 & 2,5 \\
\hline
\end{tabular}

Obs: A área fundida e penetração se referem à medidas tomadas na região abaixo da superfície da placa de teste.

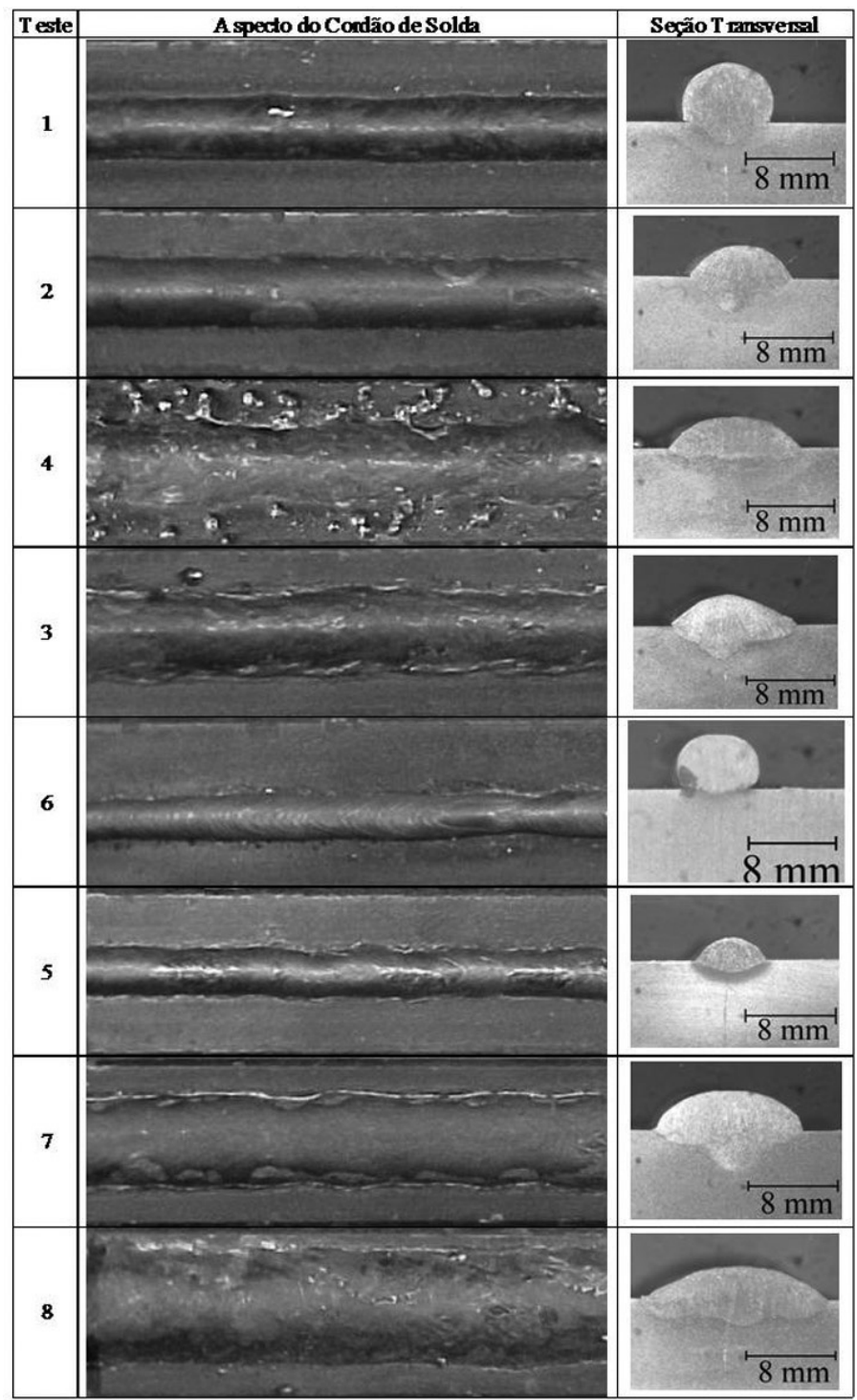

Figura 11. Aspecto e seção transversal dos cordões de solda

Agora fazendo a comparações para ver o efeito da polaridade (Teste 1 x 2 e 4 x 3 , todos à $250 \mathrm{~A}$ ), o uso de CC- com a proteção com $\mathrm{Ar}+2 \% \mathrm{O}_{2}$ (Teste $1 \times 2$ ) leva a um cordão totalmente convexo (em contraste com o uso de $\mathrm{CC}+$ ), mas com uma penetração que chega a quase $60 \%$ da penetração obtida em CC+. Por outro lado, com a proteção com $\mathrm{Ar}+18 \% \mathrm{CO}_{2}$ (Teste 4 x 3), o uso de CC- já produz cordão com convexidade aceitável, mas com aspecto ruim e baixa penetração (cerca de $34 \%$ da obtida com $\mathrm{CC}+$ ), mesmo que eliminando o formato tipo taça da penetração. As áreas fundidas são também bem maiores em CC+. Desta forma, a questão da penetração está vinculada ao tipo de gás também e não somente à polaridade, como citado na literatura (isto reduz a probabilidade do calor gerado na conexão anódica ser maior do que a catódica independentemente da polaridade, como apregoado).

Já quanto aos respingos, pode ser observada uma maior quantidade de respingos formados na polaridade negativa quando a mistura de gás continha $\mathrm{CO}_{2}$, confirmando o que é consenso entre os autores que escreveram sobre o assunto. Mas em relação à mistura contendo $\mathrm{O}_{2}$, esta grande quantidade de respingos não foi observada. Este fenômeno pode ser explicado pelo fato de que para a mistura contendo $\mathrm{CO}_{2}$ (Figura 8) o arco "dançava" em torno da gota, alterando assim a sua direção, o que não ocorria com o uso da mistura com $\mathrm{O}_{2}$ (Figura 5), onde se pode notar que as gotas tinham uma direção bem definida.

Comparando-se os Testes 1 × 6 e 2 x 5, pode-se tentar verificar a influência da corrente, já que o gás foi o mesmo $\left(\mathrm{Ar}+2 \% \mathrm{O}_{2}\right)$. Naturalmente a redução da corrente fez reduzir o volume de material depositado por unidade de comprimento do cordão e o calor imposto, além de reduzir o efeito da quantidade de movimento das gotas impingindo a poça (maior diâmetro da gota, com menor velocidade e freqüência), reduzindo a penetração, largura e área fundida. Os perfis dos cordões foram semelhantes, apenas escalarmente menores.

Para os testes realizados utilizando-se a mesma relação velocidade de alimentação/velocidade de soldagem (comparar os Testes 1 x 7 e 4 x 8), ou seja, com a mesma quantidade de material depositado por unidade de comprimento, confirmamse as mesmas tendências observadas nas comparações entre os Testes 1 x 2 e 4 x 3. Desta forma, o uso do gás de proteção com $\mathrm{Ar}+2 \% \mathrm{O}_{2}$ consegue melhorar a questão de penetração, geração de respingos e acabamento, mas não corrige a deficiência do perfil indesejável do cordão. 


\section{Conclusões}

Para as condições e parâmetros de soldagem utilizados neste trabalho, pode-se concluir que:

(1) O modo de transferência metálica em CC- é dependente do tipo de gás de proteção utilizado, mas é possível se obter transferência sem gotas repulsivas (globular e goticular), ao contrário do que classicamente é dito na literatura clássica;

(2) O alto nível de respingos em CC- também apontado pela literatura como característico só foi observado quando se utiliza a mistura com $\mathrm{Ar}+18 \% \mathrm{CO}_{2}$;

(3) Confirmou-se a característica mencionada na literatura de que há uma maior taxa de fusão em CC-, mas os resultados indicam que esta se deva mais à subida do arco pelas paredes da ponta do arame em procura por óxidos (para emissão por campo), aumentando a eficiência térmica, do que por um maior calor gerado na conexão catódica;

(4) O gás de proteção influência os parâmetros geométricos de forma diferente dependendo da polaridade usada: o $\mathrm{Ar}+2 \% \mathrm{O}_{2}$ proporciona perfil de cordão em CC- com baixa molhabilidade (formato inadequado), mas com uma certa capacidade de penetração.

\section{Agradecimentos}

Os autores gostariam de agradecer às agências $\mathrm{CNPq}$, pelo apoio financeiro na concessão de bolsas, e à FAPEMIG, pelo suporte financeiro para infra-estrutura através do Projeto TEC $604 / 2005$.

\section{Referências}

[1] AWS, Welding Handbook: Welding Process (vol II), AWS, USA, $8^{\text {th }}$ Edition, 1991, cap 4, 955 p. (ISBN 0-87171-354-3).

[2] TALKINGTON, B. S., Variable polarity gas metal arc welding. Dissertação de Mestrado, The Ohio State University. 1998. 113p.

[3] LANCASTER, J. F., The physics of welding. $2^{\text {nd }}$ ed., New York: (Oxford: Pergamom). 1986.

[4] SCOTTI, A., A review on special metal transfer modes in GMAW. Rev. Bras. de Ciências Mecânicas - RBCM, ABCM, vol XX, no. 3, Set 1998, 1998. pp. 465-478. (ISSN 0100-7386) [5] JÖNSSON, P.G.; EAGAR, T.W. \& SZEKELY, J.(1995), Heat and Metal Transfer in Gas Metal Arc welding Using Argon and Helium, Metallurgical and Materials Transactions B, v 26B, Apr 1995, pp. 383-395

[6] USHIO, M., IKEUCHI, K., TANAKA, M. \& SETO, T. Effects of shielding gas on metal transfer. Welding International, 9 (6), 1995, pp. 36-40.

[7] RHEE, S. \& KANNATEY-ASIBU Jr., E. (1991), Observation of Metal Transfer During Gas Metal Arc Welding, Conf. on Welding and Joining Processes, ASME, Atlanta, Georgia, Dec 1991, pp. 203-213.

[8] Q LIN, X LI \& S W SIMPSON Metal transfer measurements in gas metal arc welding. Journal Of Physics D: Applied Physics. 2000. pp 347-353.
[9] BÁLSAMO, P.S.S., VILARINHO, L. O., VILELA, M. \& SCOTTI, A.; Development of an Experimental Technique for Studying Metal Transfer in Welding: Synchronized Shadowgraphy, In: Int. J. for the Joining of Materials, vol 12, no. 1, 2000, The European Institute for Joining of Materials (JOM), Denmark, pp. 1-12 (ISSN 0905-6866)

[10] VILARINHO, L.O., Desenvolvimento e avaliação de um algoritmo alternativo para soldagem mig sinérgica de alumínio. Dissertação de Mestrado, Universidade Federal de Uberlândia, MG, 2000. 111p. 